\title{
THE STABILITY OF VARIETAL PERFORMANCE OVER YEARS
}

\author{
1. THE DISTRIBUTION OF SEASONAL EFFECTS FOR \\ WHEAT GRAIN YIELD
}

\author{
D. G. PEDERSON \\ Faculty of Agriculture, University of Sydney, Sydney, New South Wales, Australia
}

Received 24.i.73

\section{SUMMARY}

\begin{abstract}
A method is proposed, using wheat yield data for New South Wales as an example, for estimating the gross environmental effect of a year. For each of ten regions of the State the environmental effects for the years 1922-70 follow a lognormal distribution. The fitted curves may be used to make probability statements about the effects of particular years.

The mean yields of wheat variety trials are highly correlated with the estimated effects for a region, although the data for at least two locations are needed for a high correlation in all cases. The possible uses for estimated effects and for the fitted lognormal curves are discussed.
\end{abstract}

\section{INTRODUCtion}

IN the initial stages of a plant breeding programme a choice may be necessary between a number of potential parents, and later in the programme there may exist a number of superior strains of which the best are to be chosen for release as varieties. The usual course of action is to carry out trials with replication over time and space and to rank the genotypes according to their mean values for the characters of interest. Yates and Cochran (1938) have suggested that the average over all genotypes for a particular trial gives an indication of the prevailing environment for that trial, and that if the values for an individual genotype are plotted against the trial means then a measure is obtained of the expected genotypic response to varying environmental conditions. Finlay and Wilkinson (1963) used this analysis to investigate the adaptability of a large number of barley genotypes, and many workers have since written on the theory of the method and its practical implications (see Knight, 1970 for references).

However, it has been pointed out that the usual regression analysis is invalid because of the relationship between the independent and dependent variables (Freeman and Perkins, 1971), and to overcome this problem Fripp (1972) has proposed that one genotype should be included in a set of trials solely to assess the environmental conditions.

The present paper is concerned with assessment of the environment in a practical situation, with particular reference to trials which are repeated over years. A method is proposed by which years may be positioned on a linear scale of suitability for plant growth, and it is shown that if long-term data are available then statements can be made about the probability of reoccurrence of similar years. 


\section{The Distribution of ENVIRONMENTAL EFFEGTS}

The culture of wheat within the State of New South Wales will be used as an example. The term " environment" will be used to denote the set of circumstances occurring in a particular growth season, and there is an associated " environmental effect" which will be taken as positive in seasons which are above average and negative in relatively unfavourable seasons.

In essence, it is proposed that if a trial is carried out at a particular location then the commercial wheat yield in the immediate region should be used to gauge the effect of the season. The smallest areas for which yield data are readily available in New South Wales are the local government areas, or shires, and two clusters of five shires were chosen for study. These are listed in table 1, with the commercial wheat acreages for the 1970-71 season and

TABLE 1

The ten shires chosen for study, their wheat area for the 1970-71 season, and their approximate total areas

\begin{tabular}{lccc}
\multicolumn{1}{c}{ Shire } & Region & $\begin{array}{c}1970-71 \text { wheat area } \\
\left(10^{3} \times \text { hectares }\right)\end{array}$ & $\begin{array}{c}\text { Total area } \\
\text { (sq. kilometres) }\end{array}$ \\
Manilla & N1 & 11 & 2350 \\
Liverpool Plains & N2 & 72 & 4550 \\
Peel & N3 & 27 & 1450 \\
Tamarang & N4 & 33 & 3000 \\
Cockburn & N5 & 6 & 2750 \\
Coolamon & S1 & 42 & 2200 \\
Culcairn & S2 & 16 & 1450 \\
Narrandera & S3 & 57 & 3750 \\
Urana & S4 & 27 & 3250 \\
Lockhart & S5 & 38 & 2600
\end{tabular}

approximate total areas. The five shires situated in the Northern sector of the State, which is characterised by a predominantly summer rainfall and the culture of hard wheats, will be referred to as regions N1 to N5. The second group of five shires are situated in the Southern sector of the State, which has a predominantly winter rainfall and in which soft wheats are grown, and these will be referred to as regions S1 to S5. The two clusters of shires are approximately 350 miles apart.

Commercial wheat yields and acreages were obtained for the period 1922-70, excluding the year 1942 for which no data were available. Within each region there has been an overall increase in average grain yield over this period, due to improved agronomic practices and the release of improved varieties, and it would therefore have been incorrect to measure the effect of a year as the deviation from the 1922-70 average. Instead, it was first necessary to fit a curve to the data for each region to determine the trend in average yield over the years. A quadratic curve was first fitted and then a cubic curve, and if the additional reduction in the total variability due to including a cubic term gave an $F$-ratio greater than unity then a cubic curve was considered necessary. A similar procedure was used to determine whether a fourth-degree polynomial was needed to obtain a good fit. The resulting ten curves are shown in fig. 1.

The deviation of an observed yield from the fitted value then represents the effect of that particular year in that region. However, as a scale adjustment each deviation was divided by the corresponding fitted value so that a 
complete crop failure gives a value of -1 rather than a value which depends on the expected yield at the time. From this point the term "effect" will be used for these adjusted deviations. It may be noted that

$$
\text { observed yield }=\text { fitted yield }+ \text { deviation }
$$

so that

$$
\begin{aligned}
\frac{\text { deviation }}{\text { fitted yield }} & =\text { effect of a year } \\
& =\frac{\text { observed yield }}{\text { fitted yield }}-1 .
\end{aligned}
$$
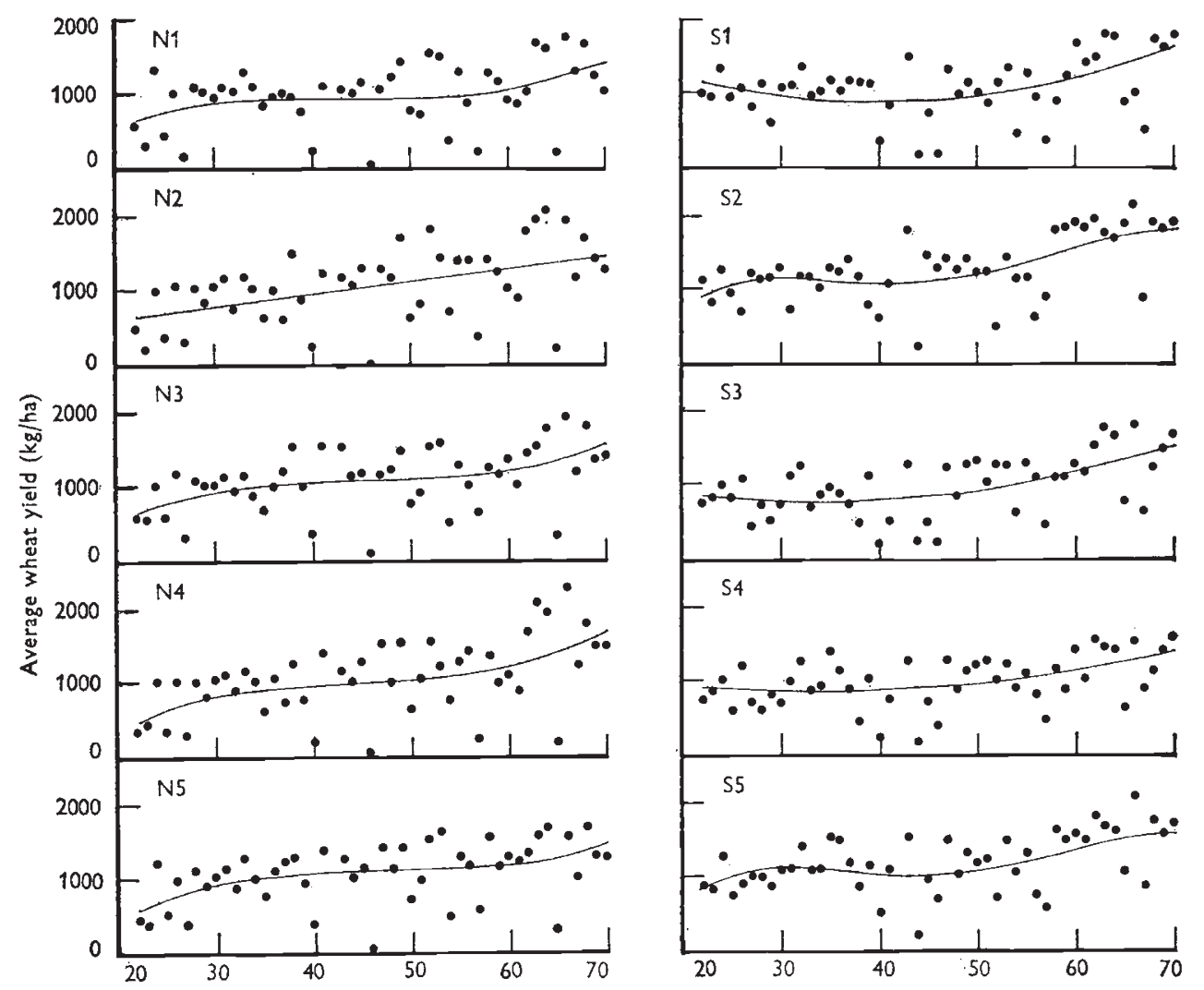

S3
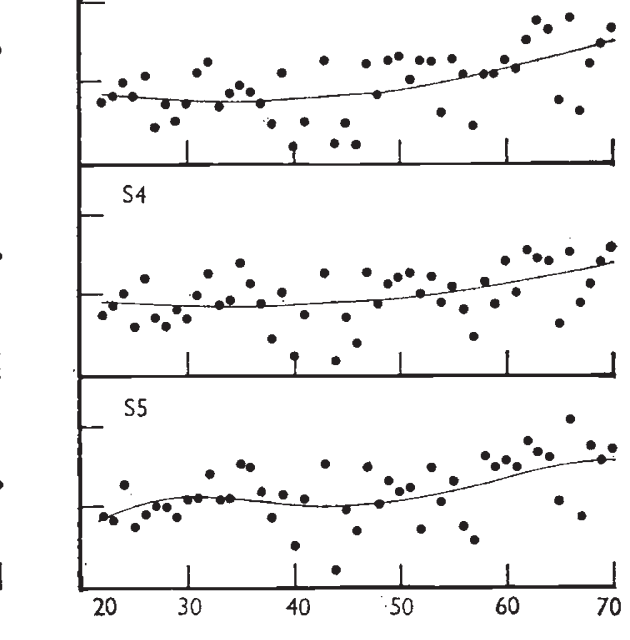

Year (19-)

Frg. 1.-Average commercial wheat yield for the ten regions of New South Wales from 1922 to 1970.

Adding unity to an estimated effect therefore gives the observed yield as a proportion of the fitted or expected yield.

From the manner in which they were determined it is clear that the set of effects for a region sums approximately to zero but this lack of independence is unavoidable. The above-diagonal section of table 2 is the matrix of correlation coefficients for the sets of effects. The Northern regions are highly correlated, with an average value of 0.90 , but for the Southern regions 
the average correlation is only $0 \cdot 68$. However, if region $\mathrm{S} 2$ is omitted then the average correlation for the remaining four regions is 0.80 , indicating that this region stands apart from the others with respect to the estimated effects. The average correlation between a Northern and Southern region is 0.41 , or 0.48 if region $\mathrm{S} 2$ is omitted.

TABLE 2

Correlations between estimated effects (above-diagonal elements) and between the estimated probabilities of more favourable years (below-diagonal elements) for the ten regions in New South Wales

\begin{tabular}{|c|c|c|c|c|c|c|c|c|c|c|c|}
\hline & \multicolumn{10}{|c|}{ Region } \\
\hline & & N1 & N2 & N3 & N4 & N5 & S1 & S2 & S3 & S4 & S5 \\
\hline & ( N1 & - & 0.89 & 0.89 & 0.88 & 0.93 & 0.57 & $0 \cdot 13$ & 0.53 & 0.44 & 0.40 \\
\hline & N2 & 0.85 & - & 0.89 & 0.95 & 0.89 & 0.54 & 0.04 & 0.48 & 0.36 & 0.30 \\
\hline & N3 & 0.85 & 0.86 & - & 0.90 & 0.93 & 0.61 & $0 \cdot 17$ & 0.49 & 0.40 & 0.39 \\
\hline & N4 & 0.83 & 0.95 & 0.86 & - & 0.90 & 0.57 & $0 \cdot 11$ & 0.55 & 0.48 & 0.42 \\
\hline & N5 & 0.88 & 0.87 & 0.92 & 0.88 & - & 0.65 & $0 \cdot 18$ & 0.53 & 0.45 & 0.45 \\
\hline & S1 & 0.49 & 0.46 & 0.55 & 0.49 & 0.59 & - & 0.51 & 0.84 & 0.76 & 0.79 \\
\hline & S2 & $0 \cdot 17$ & 0.07 & $0 \cdot 24$ & 0.14 & $0 \cdot 25$ & 0.41 & - & 0.26 & 0.42 & 0.77 \\
\hline & S3 & 0.44 & $0 \cdot 42$ & $0 \cdot 40$ & 0.47 & 0.44 & 0.78 & $0 \cdot 14$ & - & 0.87 & 0.72 \\
\hline & S4 & 0.36 & $0 \cdot 30$ & 0.34 & 0.40 & 0.39 & 0.72 & 0.31 & 0.87 & - & 0.84 \\
\hline & S5 & 0.35 & 0.35 & 0.35 & 0.34 & $0 \cdot 40$ & 0.72 & 0.68 & 0.67 & 0.80 & - \\
\hline
\end{tabular}

Fig. 2 shows the histogram of effects for each region. It was anticipated that each set of effects would follow a normal distribution but the histograms are in fact all negatively skewed. Estimated skewness and kurtosis coefficients, as defined by Bliss (1967), are shown in table 3. For a normal distribution each of these coefficients has an expected value of zero. The average for each area was obtained by first averaging the sample sums of powers of deviations from the mean and then calculating $g_{1}$ and $g_{2}$.

A lognormal distribution was fitted to the data for each region. The probability density function of the variable $e$ is

$$
\begin{aligned}
f(e) & =\frac{1}{(2 \pi)^{\frac{1}{2}} c(a-e)} \exp \left\{-\frac{1}{2}\left[\frac{1}{c} \ln \left(\frac{e-a}{b}\right)\right]^{2}\right\}, \quad e<a \\
& =0 \quad, \quad e \geqq a .
\end{aligned}
$$

A method of estimating the three parameters $a, b$, and $c$ has been given by Kendall and Stuart (1963), and the resulting fitted curves are shown in fig. 2. There is apparent agreement between observation and expectation in each case and this is confirmed by the uniformly non-significant values for the chi-squares testing goodness of fit, as given in table 4 .

Considering any one region, for a year with estimated effect $e^{*}$ we can use the fitted lognormal curve to estimate the probability of a more favourable year, viz.

$$
\text { Prob. } \begin{aligned}
\left(e>e^{*}\right)= & \frac{1}{c(2 \pi)^{\frac{1}{2}}} \int_{e^{*}}^{a} \frac{1}{a-e} \exp \left\{-\frac{1}{2}\left[\frac{1}{c} \ln \left(\frac{e-a}{b}\right)\right]^{2}\right\} d e \\
& =\frac{1}{(2 \pi)^{\frac{1}{3}}} \int_{-\infty}^{\frac{1}{c} \ln \left(\frac{e^{*}-a}{b}\right)} \exp \left(-\frac{1}{2} z^{2}\right) \cdot d z, \quad b<0,
\end{aligned}
$$




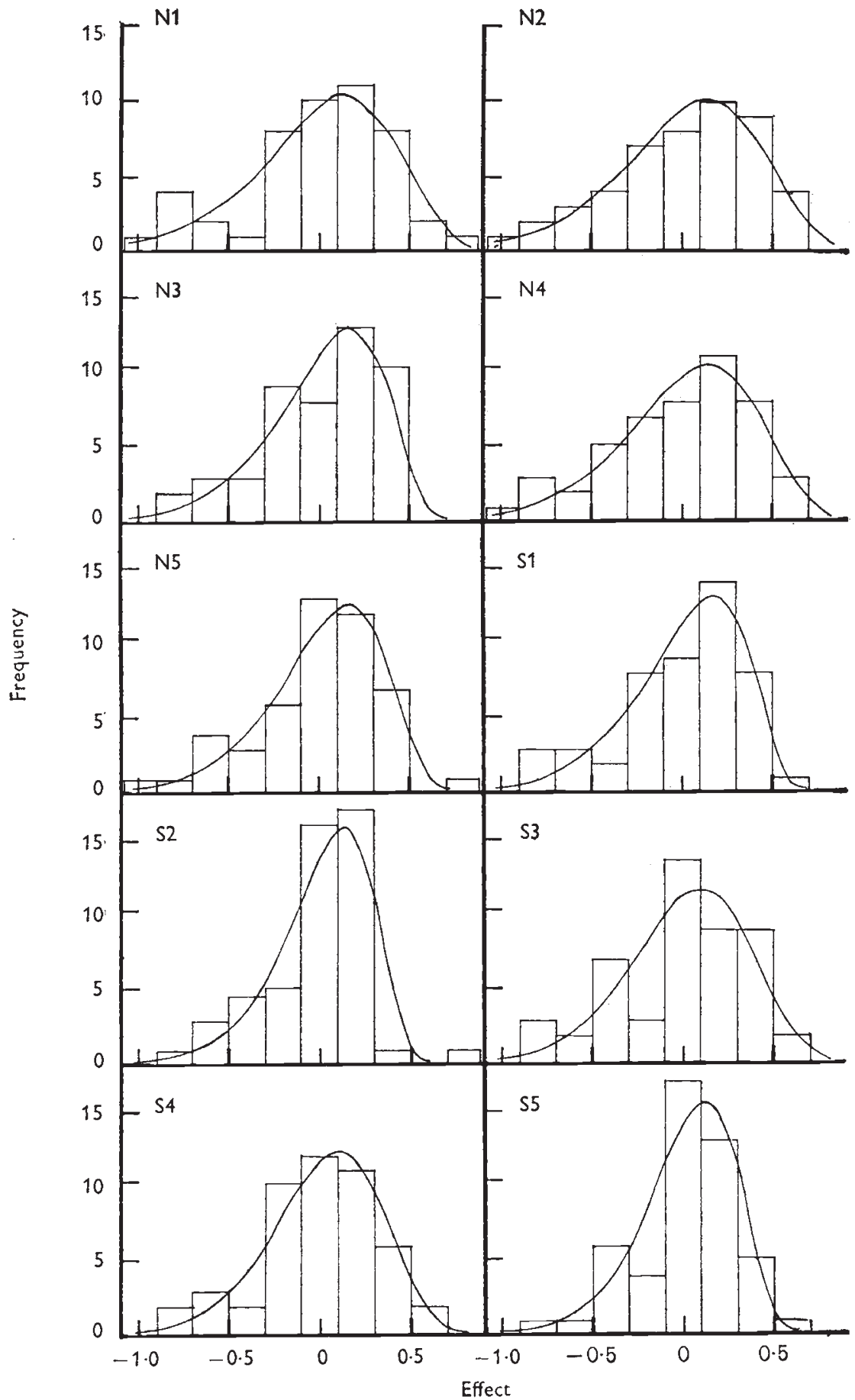

Fig. 2.-Observed distributions of seasonal effects and fitted lognormal curves for the ten regions of New South Wales. 
where $z$ is the standardised normal variate. Tables of the normal probability integral were used to evaluate the probability for each year and region. The correlations between the ten sets of probabilities, one set per region, are given in the below-diagonal section of table 2. For the Northern regions the average correlation is an agreeably high 0.88 , while for the Southern regions

TABLE 3

Measures of skewness $\left(\mathrm{g}_{1}\right)$ and kurtosis $\left(\mathrm{g}_{2}\right)$ for the distributions of wheat effects in ten regions of $\mathcal{N e w}$ South Wales

\begin{tabular}{ccr} 
Region & \multicolumn{1}{c}{$g_{1}$} & \multicolumn{1}{c}{$g_{2}$} \\
N1 & $-0.61 \pm 0.34$ & $0.15 \pm 0.67$ \\
N2 & $-0.58 \pm 0.34$ & $-0.48 \pm 0.67$ \\
N3 & $-0.81 \pm 0.34$ & $0.25 \pm 0.67$ \\
N4 & $-0.63 \pm 0.34$ & $-0.12 \pm 0.67$ \\
N5 & $-0.78 \pm 0.34$ & $0.48 \pm 0.67$ \\
average N & $-0.67 \pm 0.15$ & $0.06 \pm 0.30$ \\
S1 & $-0.90 \pm 0.34$ & $0.44 \pm 0.67$ \\
S2 & $-0.83 \pm 0.34$ & $1.37 \pm 0.67$ \\
S3 & $-0.45 \pm 0.34$ & $-0.65 \pm 0.67$ \\
S4 & $-0.51 \pm 0.34$ & $-0.04 \pm 0.67$ \\
S5 & $-0.71 \pm 0.34$ & $0.82 \pm 0.67$ \\
average S & $-0.67 \pm 0.15$ & $0.32 \pm 0.30$
\end{tabular}

\section{TABLE 4}

Chi-squares testing goodness of fit between the observed histograms of effects and fitted lognormal distributions for ten regions in New South Wales. The approximate probability associated with each chi-square is shown

$\begin{array}{cccc}\text { Region } & \text { Chi-square } & \begin{array}{c}\text { Degrees of } \\ \text { freedom }\end{array} & \text { Probability } \\ \text { N1 } & 8 \cdot 3 & 5 & 0 \cdot 15 \\ \text { N2 } & 1 \cdot 0 & 5 & 0 \cdot 95 \\ \text { N3 } & 4 \cdot 2 & 4 & 0 \cdot 40 \\ \text { N4 } & 2 \cdot 7 & 5 & 0 \cdot 75 \\ \text { N5 } & 2 \cdot 7 & 4 & 0 \cdot 60 \\ \text { S1 } & & & \\ \text { S2 } & 2 \cdot 6 & 4 & 0 \cdot 60 \\ \text { S3 } & 5 \cdot 5 & 3 & 0 \cdot 15 \\ \text { S4 } & 6 \cdot 8 & 4 & 0 \cdot 15 \\ \text { S5 } & 2 \cdot 5 & 4 & 0 \cdot 65 \\ & 4 \cdot 3 & 2 & 0.12\end{array}$

the average is 0.61 if region $\mathrm{S} 2$ is included and 0.76 if it is omitted. The average correlation between a Northern and a Southern region is 0.36 , or 0.41 if region $\mathrm{S} 2$ is omitted.

\section{Application to VARIETy trials}

Since effects have been calculated from commercial wheat yields it is possible that they have no relevance to variety trials. Only limited data were available for comparison, from trials carried out by the New South Wales Department of Agriculture. 
Within region $\mathrm{S} 3$ a trial is carried out annually at one location with two planting dates. The trial is laid out as randomised complete blocks with four blocks and a plot size of $\frac{1}{100}$ of a hectare, and there are usually 10 to 15 varieties included. For the years 1963 to 1970 the varieties Falcon, Heron, and Olympic were included in the early sowing and also in the late sowing with the exclusion of the year 1969, and in fig. 3 the mean yields for these three varieties are plotted against the estimated effects for region S3. For the early sowing the correlation between mean yield and estimated effect is 0.96 and for the late sowing the correlation is 0.95 . When the two sowings are combined the correlation is 0.99 and there is a good fit to the leastsquares regression line (fig. 3 ).

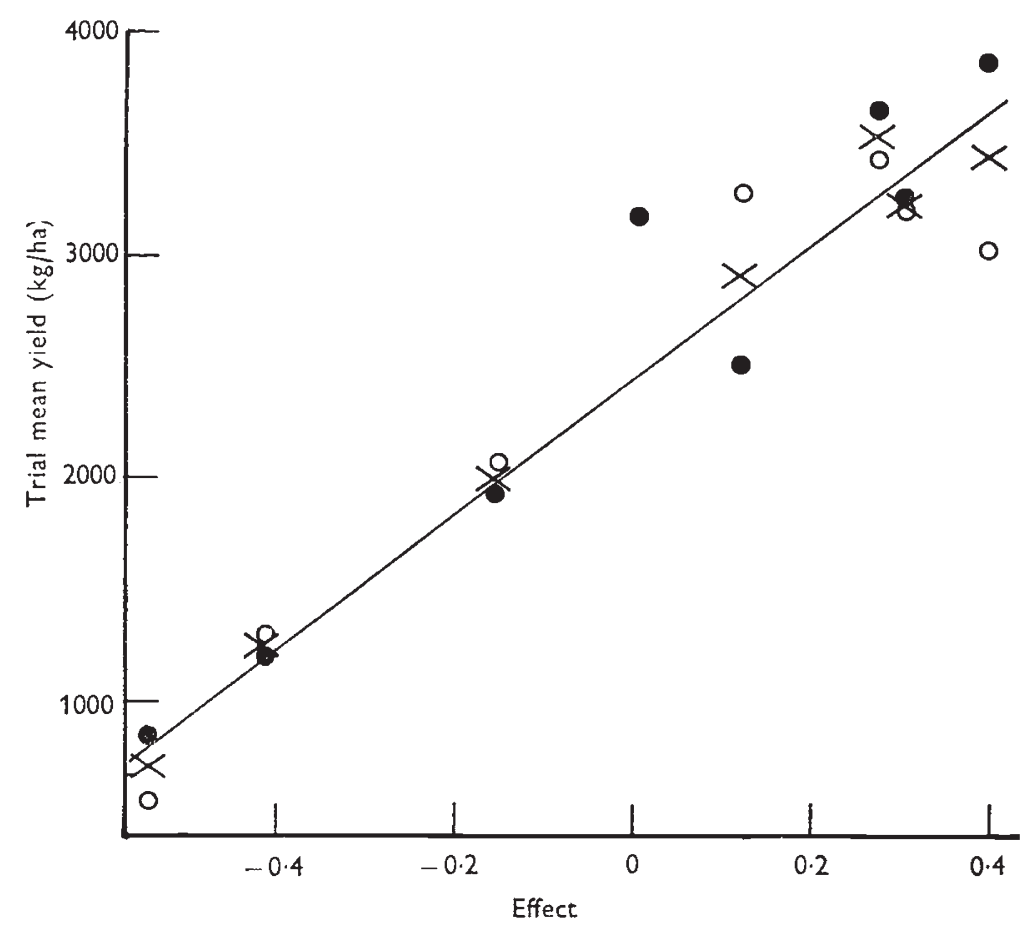

FIG. 3.-Average yield for three varieties, from a variety trial at Yanco in region S3, plotted against regional effect. ( $=$ Early sowing, $O=$ late sowing, $x=$ combined sowings.)

Trials are carried out annually at two locations in region S2. There are three or four replicates per variety per year and the plot size is from 0.007 to 0.040 of a hectare. The varieties Gamenya, Heron, and Olympic were grown at the Henty location in the years 1964 to 1970, and in fig. 4 the average annual yields are plotted against the corresponding effects for region S2. The correlation is $0 \cdot 86$. The data from the Walbundrie location, for the same varieties and years with the exception of 1965, are also plotted in fig. 4 and in this case the correlation is $0 \cdot 61$. When the data from the two locations are averaged there is a good fit to the least-squares regression line and a correlation of 0.92 .

Four other comparisons may be made between trial data and estimated effects. In each case the plot sizes and replicate numbers are the same as for 
the Henty and Walbundrie locations. At Coolamon in region S1 there were four varieties in common for 6 years and the correlation between average yield and estimated effect is 0.71 . At Booree Creek in region S5 the correlation is 0.64 for three varieties in 6 years. For the Northern regions, at Quirindi in region N4 the correlation is 0.72 for four varieties in 6 years, while at Tamworth in region $\mathrm{N} 3$ the correlation is -0.61 for three varieties in 6 years.

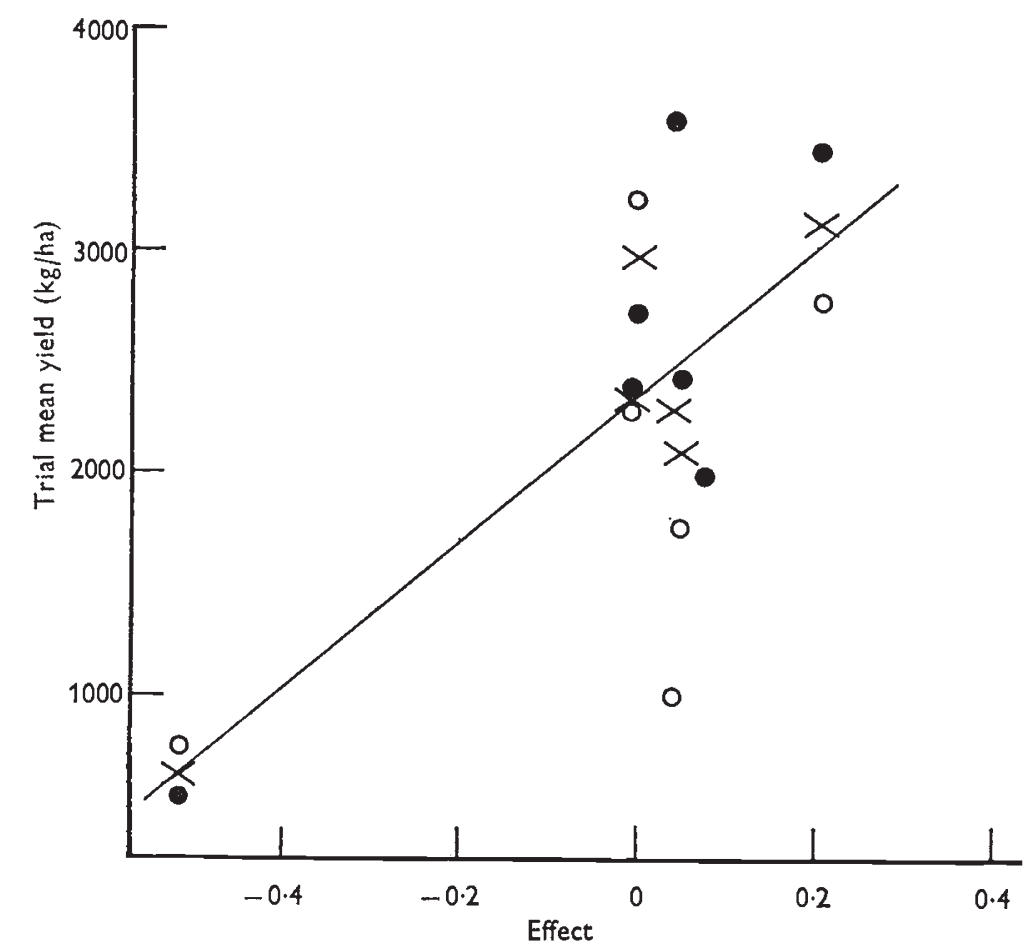

Frg. 4.-Average yield for three varieties, from two variety trials in region S2, plotted against regional effect. ( $=$ Henty data, $O=$ Walbundrie data, $x=$ combined data.)

\section{Discussion}

In a stability analysis the requirement is that the assessment of the environment should be carried out independently of the experimental trials from which genotypic means are obtained (Freeman and Perkins, 1971). Effects estimated from commercial crop yields satisfy this requirement, and it is reassuring that there is an apparent linear relationship between the estimated effects and the trial mean yields. For one set of data the correlation between these variables is 0.99 , even though the effects refer to a region of approximately 3750 sq. kilometres and the annual trial data are from 24 plots of $\frac{1}{100}$ of a hectare each. The data from the other trials indicate that at least two locations should be sampled to obtain a high correlation between effect and mean yield, presumably because of a high location $\times$ year interaction.

The high correlation between estimated effects and trial yields loses some 
of its relevance if only an approximate analysis of stability is required or if the main emphasis is on a graphical comparison of genotypic responses to varying environmental conditions. Statistical objections aside, there is no doubt that the best assessment of the environmental conditions pertaining to a trial is afforded by the trial mean yield itself. However, the import of the present paper is that only long-term data can give the distribution of effects over time. For the particular example of wheat yield in New South Wales the distribution of effects is invariably negatively skewed but there is no consistency with respect to the degree of kurtosis, and for each region a good fit is obtained to a lognormal distribution. The " average" distributions for the Northern and Southern sectors are very similar, indicating a lack of geographical and hence environmental dependence.

Determining the distribution of effects for a given region involves two stages of curve-fitting. When the lognormal curves are used to determine the probability of more favourable years for each region the average correlation between sets of probabilities is 0.88 for the Northern regions and 0.76 for the Southern regions excluding S2, indicating that the curve-fitting procedure is suitably accurate. The correlations provide only lower limits to the level of accuracy since there may well be true differences between the probabilities for adjacent regions.

A knowledge of the distribution for a particular region could be used in the subjective assessment of varietal yields obtained over several years, with less weight being given to "atypical" years than to years with a high probability of re-occurrence. There could also be an application to the problem of how many years are sufficient for testing a variety before release. It seems reasonable that 2 years of very similar effect supply less information than 2 years at opposite ends of the scale of effects, and it may be judged that sufficient testing has been carried out if the highest and lowest effects for the test years span, say, 50 per cent of the distribution of effects.

Since rainfall is probably dominant among the climatic factors which influence cereal yield within Australia, the distribution of effects may simply reflect the seasonal variation in components of rainfall. Cornish (1949, 1950) used wheat yield data for the State of South Australia to determine the relationship between yield and seasonal rainfall and concluded that a rainfall record provides a sufficiently accurate index of seasonal conditions for the particular environment considered. When different years are being compared the existence of a dominant climatic factor is desirable since it lends strength to the inference that two seasons of the same effect are environmentally identical.

Acknowledgments. - The author is grateful to the Division of Plant Industry of the New South Wales Department of Agriculture for permission to use the data from their annual wheat variety trials.

\section{REFERENCES}

BLIss, c. I. 1967. Statistics in Biology, Volume 1. McGraw-Hill, New York.

CORNish, E. A. 1949. Yield trends in the wheat belt of South Australia during 1896-1941. Aust. 7. Sci. Res., series B, 2, 83-137.

CORNisH, E. A. 1950. The influence of rainfall on the yield of wheat in South Australia. Aust. F. Sci. Res., series B, 3, 178-218.

FINLAY, K. W., AND WILKINSON, G. N. 1963. The analysis of adaptation in a plant breeding programme. Aust. F. Agr. Res., 6, 742-754. 
FREEMAN, G. H., AND PERKINS, JEAN M. 1971. Environmental and genotype-environmental components of variability. VIII. Relations between genotypes grown in different environments and measures of these environments. Heredity, 27, 15-23.

FRIPP, YVONNE. 1972. Genotype-environmental interactions in Schizophyllum commune. II. Assessing the environment. Heredity, 28, 223-238.

kendali, M. G., AND stuart, A. 1963. The Advanced Theory of Statistics. Volume 1, Distribution Theory. Charles Griffin, London.

KNIGHT, R. 1970. The measurement and interpretation of genotype-environment interactions. Euphytica, 19, 225-235.

yates, f., and cochran, w. G. 1938. The analysis of groups of experiments. F. Agric. Sci., $28,556-580$. 